\title{
Prognostic value of KRAS mutation status in colorectal cancer patients: A population-based competing risk analysis
}

\author{
Dongjun Dai ${ }^{1}$, Yanmei Wang ${ }^{1}$, Liyuan Zhu ${ }^{2}$, Hongchuan Jin ${ }^{2}$, Xian Wang ${ }^{\text {Corresp. } 1}$ \\ ${ }^{1}$ Department of Medical Oncology, Sir Run Run Shaw Hospital, Medical School of Zhejiang University, Zhejiang University, Hangzhou, Zhejiang, China \\ 2 Laboratory of Cancer Biology, Key Lab of Biotherapy, Sir Run Run Shaw Hospital, Medical School of Zhejiang University, Zhejiang University, Hangzhou, \\ Zhejiang, China
}

Corresponding Author: Xian Wang

Email address: wangx118@zju.edu.cn

Background: To using competing analyses to estimate the prognostic value of KRAS mutation in colorectal cancer (CRC) patients and to build nomogram for CRC patients who had KRAS testing.

Method: The cohort was selected from the Surveillance, Epidemiology, and End Results (SEER) database. Cumulative incidence function (CIF) model and multivariate Fine-Gray regression for proportional hazards modeling of the subdistribution hazard (SH) model were used to estimate the prognosis. A SH model based nomogram was built after a variable selection process. The validation of the nomogram was conducted by discrimination and calibration with 1,000 bootstraps.

Results: We included 8,983 CRC patients who had KRAS testing. SH model found that KRAS mutant (MT) patients had worse CSS than KRAS wild type (WT) patients in overall cohort $(\mathrm{HR}=1.10,95 \% \mathrm{Cl}=$ 1.04-1.17, $\mathrm{p}<0.05)$, and in subgroups that comprised stage III CRC ( $\mathrm{HR}=1.28,95 \% \mathrm{Cl}=1.09-1.49, \mathrm{p}<$ $0.05)$ and stage IV CRC $(H R=1.14,95 \% \mathrm{Cl}=1.06-1.23, \mathrm{p}<0.05)$, left side colon cancer $(\mathrm{HR}=1.28$, $95 \% \mathrm{Cl}=1.15-1.42, \mathrm{p}<0.05)$ and rectal cancer $(\mathrm{HR}=1.23,95 \% \mathrm{Cl}=1.07-1.43, \mathrm{p}<0.05)$. We built the $\mathrm{SH}$ model based nomogram, which showed well accuracy by internal validation of discrimination and calibration. Calibration curves represented good agreement between the nomogram predicted CRC caused death and actual observed CRC caused death. The time dependent area under the curve ofreceiver operating characteristic curves (AUC) was over 0.75 for the nomogram.

Conclusion: This is the first population based competing risk study on the association between KRAS mutation status and the CRC prognosis. The mutation of KRAS indicated a poor prognosis of CRC patients. The current competing risk nomogram would help physicians to predict cancer specific death of CRC patients who had KRAS testing. 


\section{Prognostic Value Of KRAS Mutation Status In}

2 Colorectal Cancer Patients: A Population-based

3 Competing Risk Analysis

Dongjun Dai ${ }^{1}$, Yanmei Wang ${ }^{1}$, Liyuan Zhu², Hongchuan $\mathrm{Jin}^{2}$, Xian Wang ${ }^{1, *}$

${ }^{1}$ Department of Medical Oncology, Sir Run Run Shaw Hospital, Medical School of Zhejiang University, Hangzhou, China; '2Laboratory of Cancer Biology, Key Lab of Biotherapy, Sir Run Run Shaw Hospital, Medical School of Zhejiang University, Hangzhou, China

${ }^{*}$ Correspondence: Dr. Xian Wang, email: wangx118@zju.edu.cn, Tel/Fax: +86 5718600 6929

\section{Abstract}

Background: To using competing analyses to estimate the prognostic value of KRAS mutation in colorectal cancer (CRC) patients and to build nomogram for CRC patients who had KRAS testing.

Method: The cohort was selected from the Surveillance, Epidemiology, and End Results (SEER) database. Cumulative incidence function (CIF) model and multivariate Fine-Gray regression for proportional hazards modeling of the subdistribution hazard $(\mathrm{SH})$ model were used to estimate the prognosis. A SH model based nomogram was built after a variable selection process. The validation of the nomogram was conducted by discrimination and calibration with 1,000 bootstraps.

Results: We included 8,983 CRC patients who had KRAS testing. SH model found that KRAS mutant (MT) patients had worse CSS than KRAS wild type (WT) patients in overall cohort $(\mathrm{HR}=1.10,95 \% \mathrm{Cl}=1.04-1.17, \mathrm{p}<0.05)$, and in subgroups that comprised stage III CRC $(\mathrm{HR}=1.28,95 \% \mathrm{Cl}=1.09-1.49, \mathrm{p}<0.05)$ and stage IV CRC $(\mathrm{HR}=1.14,95 \% \mathrm{Cl}$ $=1.06-1.23, \mathrm{p}<0.05)$, left side colon cancer $(\mathrm{HR}=1.28,95 \% \mathrm{Cl}=1.15-1.42, \mathrm{p}<0.05)$ and rectal cancer $(\mathrm{HR}=1.23,95 \% \mathrm{Cl}=1.07-1.43, \mathrm{p}<0.05)$. We built the $\mathrm{SH}$ model based nomogram, which showed well accuracy by internal validation of discrimination and calibration. Calibration curves represented good agreement between the nomogram predicted CRC caused death and actual observed CRC caused death. The time 
dependent area under the curve of receiver operating characteristic curves (AUC) was over 0.75 for the nomogram.

Conclusion: This is the first population based competing risk study on the association between KRAS mutation status and the CRC prognosis. The mutation of KRAS indicated a poor prognosis of CRC patients. The current competing risk nomogram would help physicians to predict cancer specific death of CRC patients who had KRAS testing.

Keywords: KRAS, colorectal cancer, nomograms, prognosis, SEER, competing risk

\section{Introduction}

Colorectal cancer (CRC) is the second and third most common cancer of women and men worldwide, respectively (Bray et al. 2018). The amount of deaths due to CRC ranked the second among all cancer types in 2018(Bray et al. 2018). CRC is a heterogeneous disease with various genetic events(Inamura 2018; Punt et al. 2017). Target therapy such as anti-epidermal growth factor receptor (EGFR) therapy has been developed for metastatic CRC(Chan et al. 2017).

KRAS is an effector molecule that makes the signal transduction from ligand-bound EGFR to the nucleus(Liu et al. 2019). KRAS has intrinsic GTPase activity and it binds to GTP to active downstream pathway, such as RAS/RAF/MAPK and PI3K/AKT pathways, to promote cell proliferation. Normally, the GTPase activating proteins (GAPs) would enhance the GTPase activity of KRAS and transform the status of GTP-bound KRAS into a status of GDP-bound KRAS status, terminating the downstream signaling. However, some types of KRAS mutation could impair the GAP binding to KRAS and lead to a continuous GTP-bound KRAS status to promote the proliferation related pathways and cancer development(Cox \& Der 2010). The mutation of KRAS would also impair the efficacy of EGFR-targeted therapy(Liu et al. 2019). KRAS mutation is found in about $33 \%$ to $45 \%$ of CRC(Tan \& Du 2012). Hence, the KRAS testing is recommended for CRC patients who would receive anti-EGFR therapy. The anti-EGFR therapy is limited to KRAS wild type (WT) CRC patients but not KRAS mutant (MT) patients(Markman et al. 2010).

Despite the KRAS mutation status is a biomarker for the anti-EGFR therapy of CRC patients, whether it is an independent prognostic factor in CRC was controversial. In metastatic CRC, there were studies showed that KRAS MT patients had worse progression-free survival (PFS)(Modest et al. 2016; Souglakos et al. 2009) or overall survival (OS)(Modest et al. 2016) than KRAS WT patients, while other study found there was no association between KRAS mutation status and PFS or OS of CRC patients(Kim 
et al. 2016). Among stage II and III CRC, there were studies found KRAS mutation would worsen the OS(Richman et al. 2009) or disease-free survival (DFS)(Deng et al. 2015; Lee et al. 2015) of patients while other study found KRAS mutation was not associated with the OS or recurrence-free survival (RFS) of CRC patients(Roth et al. 2010). In stage III colon cancer, a study found KRAS mutational status was not associated with the OS or RFS or DFS of patients(Ogino et al. 2009), while more recently studies found the KRAS mutation would worsen the DFS(Sperlich et al. 2018) or survival after recurrence (SAR)(Taieb et al. 2019) of patients. To be noted, most of these studies included with limited amount of CRC patients who had KRAS testing.

Surveillance, Epidemiology, and End Results (SEER) database of the National Cancer Institute is a national collaboration program of Unite States, covering $34.6 \%$ of the national population. It collects the incidence, survival and treatment data of cancer patients. There was a SEER based study(Charlton et al. 2017) on the association between KRAS mutation status and the OS of patients with left or right side CRC. However, despite CRC is an aggressive disease, the median age at diagnosis for colon cancer patients is 68 years in men and 72 years in women, respectively; for rectal cancer patients it is 63 years in both men and women(Society. 2017). In this case, the competing risk events might be involved, as the elders might dead from diseases other than CRC such as cardiovascular disease(Zhang 2017). Competing risk models such as the cumulative incidence function (CIF) model and Fine-Gray regression for proportional hazards modeling of the subdistribution hazard (SH) model (Austin et al. 2016) should be used for the prognostic analyses of population based studies of CRC.

Nomogram is a useful method to predict the probability of patients' clinical outcomes(Balachandran et al. 2015). It has compared favorably to traditional TNM staging systems in the prognostic prediction in a series of cancers(Bobdey et al. 2018; He et al. 2018). To our knowledge, there is currently no nomogram constructed for predicting the outcomes of CRC patients who had KRAS testing.

Here we performed a SEER based study to evaluate the association between KRAS mutation status and the cancer specific survival (CSS) of CRC patients by using competing risk analyses. We also drew a SH model based nomogram for the cancer specific death prediction of CRC patients who had KRAS testing.

\section{Methods}

\section{Cohort information}


The SEER based cohort was selected using SEER*Stat 8.3.5 software (SEER ID: daid). The access to Collaborative Stage Site-Specific Factor 9 (KRAS mutation status) was granted by the National Cancer Institute $(\mathrm{NCl})$. We included patients who met the inclusion criteria as the follows: (1) it should be a CRC patient who had KRAS testing; (2) it should include sufficient clinicopathological information of the variables in current study (Listed in Table 1). As the information of KRAS testing was collected since 2010, we only included patients who were diagnosed equal to or after 2010. Finally, as shown in Fig. $S 1$, to find an adequate follow-up time, the patients diagnosed between 2010-2012 were included. For tumor location, left side means the tumors in splenic flexure, descending colon, sigmoid and rectosigmoid junction, and right side means the tumors in cecum, ascending colon, hepatic flexure and transverse. We defined the median follow-up as the median observed survival time. The last follow-up time was December 31, 2015.

\section{Statistical analyses}

The chi-square test was applied for the comparisons of difference variables between KRAS WT and KRAS MT CRC patients. The cumulative incidences of death (CID) was estimated for cancer related deaths and non-cancer related deaths. Multivariate SH model, which involved all variables, was used to assess the CSS of CRC patients. SH model based nomogram was constructed to predict the 1-year, 2-year and 3-year CSS of CRC patients who had KRAS testing. To be noted, many prediction factors in one model might cause over-fitting. Hence, we used the variable selection to improve the interpretation and the accuracy of prediction of the competing nomogram(Ha et al. 2014). Penalized variable selection was performed by using methods of least absolute shrinkage and selection operator (LASSO), measure-correlate-predict (MCP) and smoothly clipped absolute deviation (SCAD) to select variables for $\mathrm{SH}$ model based nomogram. This nomogram was internally validated by discrimination and calibration with 1,000 times bootstraps (Balachandran et al. 2015). The calibration curves and the area under the curve of receiver operating characteristic curve (AUC) were used for discrimination and calibration, respectively.

The statistical analyses of current study were performed by a series of packages in $R$ version 3.5.1. The detailed using of those packages could be found in our previous published study(Dai et al. 2020). We considered a p-value less than 0.05 as statistically significant.

\section{Results}




\section{Cohort information}

As shown in Table 1, there were totally 8,983 CRC patients (3,616 KRAS MT patients and 5,367 KRAS WT patients) included in current study. Significant differences were found between KRAS MT and KRAS WT patients among variables of age, race, location, surgery, tumor stage, grade, positive regional nodes amount, and chemotherapy experience $(p<0.05)$. In detail, compared with KRAS WT patients, the KRAS MT patients had more African American race (14.33\% vs. $11.14 \%$ ), more occurrence in right side of the colon $(45.52 \%$ vs. $36.28 \%)$, less surgery performance $(77.43 \%$ vs. $79.90 \%)$, more metastatic site $(55.86 \%$ vs. $48.72 \%)$, lower grade (grade III \& IV: $19.69 \%$ vs. $25.53 \%)$, and more chemotherapy experience $(74.45 \%$ vs. $71.42 \%)$. The median follow-time were 30 months and 36 months for KRAS MT and KRAS WT, respectively. In KRAS MT patients, the death rate caused by cancer and other reasons were $49.89 \%$ and $13.69 \%$, respectively. In KRAS WT patients, the death rate caused by cancer and other reasons were $42.59 \%$ and $14.83 \%$, respectively.

\section{KRAS MT patients had worse outcomes than KRAS WT patients}

The CIF plots showed that the KRAS MT patients had a worse CSS than KRAS WT patients ( $p<0.001$, Figure 1a). We further performed subgroup analysis of KRAS mutation status among different AJCC 7th stages and tumor locations. The CIF plots found that KRAS mutation had no association with the CSS of stage I ( $p=0.347$, Figure $1 b)$ and stage II ( $p=0.093$, Figure 1c) CRC patients while it contributed to worse CSS in stage III ( $p=0.009$, Figure $1 d)$ and stage IV ( $p=0.0013$, Figure 1e) CRC patients. In addition, the CIF plots showed that KRAS mutation was a hazard factor for the CSS of patients with cancers in the location of left colon, right colon or rectum $(p<0.001$, Figure 2).

As shown in Table 2, the multivariate SH model showed that KRAS MT patients had worse CSS $(\mathrm{HR}$ [Hazard ratio] $=1.10,95 \% \mathrm{Cl}[95 \%$ confdence index $]=1.04-1.17, \mathrm{p}<$ $0.05)$ than KRAS WT patients. Further subgroup analysis found the KRAS mutation was an independent risk factor for the CSS of stage III $(\mathrm{HR}=1.28,95 \% \mathrm{Cl}=1.09-1.49, \mathrm{p}<$ $0.05)$ and stage IV $(\mathrm{HR}=1.14,95 \% \mathrm{Cl}=1.06-1.23, \mathrm{p}<0.05) \mathrm{CRC}$ patients. Moreover, we found KRAS shorten the CSS in patients with cancers occurred at left colon (HR = $1.28,95 \% \mathrm{Cl}=1.15-1.42, \mathrm{p}<0.05)$ or rectum $(\mathrm{HR}=1.23,95 \% \mathrm{Cl}=1.07-1.43, \mathrm{p}<0.05)$ but not right colon $(\mathrm{HR}=1.07,95 \% \mathrm{Cl}=0.97-1.19, \mathrm{p}>0.05)$.

\section{Multivariate SH analyses of each variable for the CSS of KRAS MT and KRAS WT CRC patients}


As shown in Table 3, the multivariate $\mathrm{SH}$ model identified the absence of surgery, higher tumor stage and grade, and unmarried status as risk factors for both KRAS MT and KRAS WT CRC patients $(H R>1, P<0.05)$. We observed there was no significant association between sex and the prognosis in neither KRAS MT nor KRAS WT CRC patients $(p>0.05)$.

Prognostic discrepancies were found in other variables between KRAS MT and WT CRC patients. The older age was a protective factor for KRAS MT patients (HR $<1, P<$ $0.05)$ but was not associated with the prognosis of KRAS WT patients $(p>0.05)$. We found that the race of African American was a risk factor for KRAS MT patients but not for KRAS WT patients. The right side colon cancer was observed to have worse CSS than left side colon cancer in KRAS WT patients $(H R>1, P<0.05)$ but not in KRAS MT patients $(p>0.05)$. Moreover, we found that the chemotherapy was only a protective factor for KRAS MT patients (HR $<1, P<0.05)$ but not for KRAS WT patients $(p>0.05)$.

\section{Nomogram construction and validation}

The LASSO, SCAD and MCP analyses all selected age, location, tumor size and stage, regional positive nodes amount, KRAS mutation status, chemotherapy experience and radiotherapy experience as the key prognostic variables of our nomogram (Table 4). These variables were then used to construct the multivariate $\mathrm{SH}$ model based nomogram to predict the 1-year, 2-year and 3-year CRC specific death (Figure 3). Internal validation showed good calibration (Figure 4a-c, there were good agreements between the nomogram predicted $\mathrm{CRC}$ death and actual observed CRC death) and discrimination (AUC $>0.75$, Figure $4 d$ ) of current nomogram.

\section{Discussion}

The KRAS testing for metastatic CRC patients was recommended by the National Comprehensive Cancer Network (NCCN). The rate of KRAS testing for metastatic or nonmetastatic CRC patients was increased in recent years according to SEER database (Fig. S2). However, the association between KRAS mutation status and the prognosis of CRC patients remains unclear. A SEER based study(Charlton et al. 2017) found that there was no association between KRAS mutation status and the OS of CRC. This might be a result of the limited follow up time, as they included the 2010-2012 data meanwhile had a last follow-up time of December 2013. Compared with this study, we included with 2010-2012 data while the last follow-up time was December 2015. The median survival time of our cohort was 33 months and the overall death rate of current study was $62.1 \%$, indicating 
209 that our follow up time was relative sufficient. Furthermore, CRC patients were often

210

211

212

213

214

215

216

217

218

219

220

221

222

223

224

225

226

227

228

229

230

231

232

233

234

235

236

237

238

239

240

241

242

243

244 diagnosed at an old age, therefore, competing risk analysis was more appropriate in the SEER based study. Our competing risk model found KRAS MT would shorten the CSS in CRC patients. Further subgroup analysis found that KRAS MT patients had worse CSS than KRAS WT patients among stage III or stage IV CRC patients or patients with left side colon cancer or rectal cancer. Moreover, the current study firstly built a competing nomogram for CRC patients who had KRAS testing.

Age was observed as a risk factor for the OS of CRC patients(Charlton et al. 2017; van Eeghen et al. 2015). However, CRC patients are usually elders who might have high potential risk of deaths from other diseases. Our competing risk model found the older age was not associated with worse CSS of CRC patients. Moreover, older KRAS MT patients might have better CSS than young patients.

Left colon cancer was found to be more sensitive to anti-EGFR targeted therapy than right colon cancer(Venook et al. 2017). The right side colon cancer was found to have more BRAF mutation than left side colon cancer, which might cause the resistant to antiEGFR therapy(van Brummelen et al. 2017) and worsen the prognosis(Salem et al. 2017). Hence, for left-sided colon cancer, KRAS WT CRC patients are more likely to be benefit from anti-EGFR targeted therapy and have better outcomes than KRAS MT patients. Indeed, we found KRAS mutation was an independent risk factor for left side colon cancer but not right side colon cancer. Moreover, in KRAS WT patients, we found right colon cancer had worse CSS than left side colon cancer meanwhile in KRAS MT patients, there was no significant prognostic difference between right and left side colon cancers.

We built a SH model-based nomogram to predict the probability of cancer specific death after a variable selection. Our nomogram was well validated. The predictors of current nomogram were easy to be obtained in clinical use. The increasing concern about competing risk had promoted researchers to develop competing risk nomograms for a groups of cancers(Brockman et al. 2015; Kattan et al. 2003; Kutikov et al. 2010; Shen et al. 2016; Yang et al. 2013).

There were certain limitations in our study. First, prognostic differences were found between KRAS codon 12 and codon 13 mutations(Imamura et al. 2012). However, the detailed KRAS mutation pattern was not registered in SEER. The detailed anti-EGFR therapy and chemotherapy strategy were also missed. Second, other genetic variables, such as BRAF mutation and microsatellite instability (MSI), were also frequently occurred in CRC and associated with the prognosis of CRC(Jung et al. 2016; Sanz-Garcia et al. 2017). These data were also not available in SEER. Third, selection bias might exist in current study as we only included patients with complete information of included 
245

246

247

248

249

250

251

252

253

254

255

256

257

258

259

260

261

262

263

264

265

266

267

268

269

270

271

272

273

274

275

276

277

278

279

variables.

\section{Conclusion}

This is the first population based competing risk study on the association between KRAS mutation status and the CRC prognosis. We found KRAS mutation would worsen the CSS for patients with stage III and stage IV CRC, and for patients with cancers in the locations of left side colon and rectum. SH based nomogram with good discrimination and calibration was constructed, which might help the clinicians to predict the 1-year, 2-year and 3-year cancer specific death of CRC patients who had KRAS testing.

\section{Acknowledgements}

This grant was supported by National Natural Science Foundation of China (81372178; 81502386; 81772944; 81572715), High level health innovative talents program in Zhejiang and Natural Science Foundation of Zhejiang (LZ17H60003).

\section{References}

Austin PC, Lee DS, and Fine JP. 2016. Introduction to the Analysis of Survival Data in the Presence of Competing Risks. Circulation 133:601-609. 10.1161/CIRCULATIONAHA.115.017719

Balachandran VP, Gonen M, Smith JJ, and DeMatteo RP. 2015. Nomograms in oncology: more than meets the eye. Lancet Oncol 16:e173-180. 10.1016/S1470-2045(14)71116-7

Bobdey S, Mair M, Nair S, Nair D, Balasubramaniam G, and Chaturvedi P. 2018. A Nomogram based prognostic score that is superior to conventional TNM staging in predicting outcome of surgically treated T4 buccal mucosa cancer: Time to think beyond TNM. Oral Oncol 81:10-15. 10.1016/j.oraloncology.2018.04.002

Bray F, Ferlay J, Soerjomataram I, Siegel RL, Torre LA, and Jemal A. 2018. Global cancer statistics 2018: GLOBOCAN estimates of incidence and mortality worldwide for 36 cancers in 185 countries. CA Cancer J Clin 68:394424. 10.3322/caac.21492

Brockman JA, Alanee S, Vickers AJ, Scardino PT, Wood DP, Kibel AS, Lin DW, Bianco FJ, Jr., Rabah DM, Klein EA, Ciezki JP, Gao T, Kattan MW, and Stephenson AJ. 2015. Nomogram Predicting Prostate Cancer-specific Mortality for Men with Biochemical Recurrence After Radical Prostatectomy. Eur Urol 67:1160-1167. 10.1016/j.eururo.2014.09.019

Chan DLH, Segelov E, Wong RS, Smith A, Herbertson RA, Li BT, Tebbutt N, Price T, and Pavlakis N. 2017. Epidermal growth factor receptor (EGFR) inhibitors for metastatic colorectal cancer. Cochrane Database Syst Rev 6:CD007047. 10.1002/14651858.CD007047.pub2

Charlton ME, Kahl AR, Greenbaum AA, Karlitz JJ, Lin C, Lynch CF, and Chen VW. 2017. KRAS Testing, Tumor Location, and Survival in Patients With Stage IV Colorectal Cancer: SEER 2010-2013. J Nat/ Compr Canc Netw 15:1484- 
1493. 10.6004/jnccn.2017.7011

Cox AD, and Der CJ. 2010. Ras history: The saga continues. Small GTPases 1:2-27. 10.4161/sgtp.1.1.12178

Dai D, Shi R, Wang Z, Zhong Y, Shin VY, Jin H, and Wang X. 2020. Competing Risk Analyses of Medullary Carcinoma of Breast in Comparison to Infiltrating Ductal Carcinoma. Sci Rep 10:560. 10.1038/s41598-019-57168-2

Deng Y, Wang L, Tan S, Kim GP, Dou R, Chen D, Cai Y, Fu X, Zhu J, and Wang J. 2015. KRAS as a predictor of poor prognosis and benefit from postoperative FOLFOX chemotherapy in patients with stage II and III colorectal cancer. Mol Oncol 9:1341-1347. 10.1016/j.molonc.2015.03.006

Ha ID, Lee M, Oh S, Jeong JH, Sylvester R, and Lee Y. 2014. Variable selection in subdistribution hazard frailty models with competing risks data. Stat Med 33:4590-4604. 10.1002/sim.6257

He C, Zhang Y, Cai Z, Duan F, Lin X, and Li S. 2018. Nomogram to Predict Cancer-Specific Survival in Patients with Pancreatic Acinar Cell Carcinoma: A Competing Risk Analysis. J Cancer 9:4117-4127. 10.7150/jca.26936

Imamura Y, Morikawa T, Liao X, Lochhead P, Kuchiba A, Yamauchi M, Qian ZR, Nishihara R, Meyerhardt JA, Haigis KM, Fuchs CS, and Ogino S. 2012. Specific mutations in KRAS codons 12 and 13, and patient prognosis in 1075 BRAF wild-type colorectal cancers. Clin Cancer Res 18:4753-4763. 10.1158/1078-0432.CCR-11-3210

Inamura K. 2018. Colorectal Cancers: An Update on Their Molecular Pathology. Cancers (Basel) 10. $10.3390 /$ cancers 10010026

Jung SH, Kim SH, and Kim JH. 2016. Prognostic Impact of Microsatellite Instability in Colorectal Cancer Presenting With Mucinous, Signet-Ring, and Poorly Differentiated Cells. Ann Coloproctol 32:58-65. 10.3393/ac.2016.32.2.58

Kattan MW, Heller G, and Brennan MF. 2003. A competing-risks nomogram for sarcoma-specific death following local recurrence. Stat Med 22:3515-3525. 10.1002/sim.1574

Kim HS, Heo JS, Lee J, Lee JY, Lee MY, Lim SH, Lee WY, Kim SH, Park YA, Cho YB, Yun SH, Kim ST, Park JO, Lim HY, Choi YS, Kwon WI, Kim HC, and Park YS. 2016. The impact of KRAS mutations on prognosis in surgically resected colorectal cancer patients with liver and lung metastases: a retrospective analysis. BMC Cancer 16:120. 10.1186/s12885-016-2141-4

Kutikov A, Egleston BL, Wong YN, and Uzzo RG. 2010. Evaluating overall survival and competing risks of death in patients with localized renal cell carcinoma using a comprehensive nomogram. J Clin Oncol 28:311-317. 10.1200/JCO.2009.22.4816

Lee DW, Kim KJ, Han SW, Lee HJ, Rhee YY, Bae JM, Cho NY, Lee KH, Kim TY, Oh DY, Im SA, Bang YJ, Jeong SY, Park KJ, Park JG, and Kang GH. 2015. KRAS mutation is associated with worse prognosis in stage III or high-risk stage II colon cancer patients treated with adjuvant FOLFOX. Ann Surg Oncol 22:187-194. 10.1245/s10434-0143826-z

Liu P, Wang Y, and Li X. 2019. Targeting the untargetable KRAS in cancer therapy. Acta Pharm Sin B 9:871-879. 10.1016/j.apsb.2019.03.002

Markman B, Javier Ramos F, Capdevila J, and Tabernero J. 2010. EGFR and KRAS in colorectal cancer. Adv Clin Chem 51:71-119.

Modest DP, Ricard I, Heinemann V, Hegewisch-Becker S, Schmiegel W, Porschen R, Stintzing S, Graeven U, Arnold D, von Weikersthal LF, Giessen-Jung C, Stahler A, Schmoll HJ, Jung A, Kirchner T, Tannapfel A, and ReinacherSchick A. 2016. Outcome according to KRAS-, NRAS- and BRAF-mutation as well as KRAS mutation variants: pooled analysis of five randomized trials in metastatic colorectal cancer by the AlO colorectal cancer study group. Ann Oncol 27:1746-1753. 10.1093/annonc/mdw261 
Ogino S, Meyerhardt JA, Irahara N, Niedzwiecki D, Hollis D, Saltz LB, Mayer RJ, Schaefer P, Whittom R, Hantel A, Benson AB, 3rd, Goldberg RM, Bertagnolli MM, and Fuchs CS. 2009. KRAS mutation in stage III colon cancer and clinical outcome following intergroup trial CALGB 89803. Clin Cancer Res 15:7322-7329. 10.1158/10780432.CCR-09-1570

Punt CJ, Koopman M, and Vermeulen L. 2017. From tumour heterogeneity to advances in precision treatment of colorectal cancer. Nat Rev Clin Oncol 14:235-246. 10.1038/nrclinonc.2016.171

Richman SD, Seymour MT, Chambers P, Elliott F, Daly CL, Meade AM, Taylor G, Barrett JH, and Quirke P. 2009. KRAS and BRAF mutations in advanced colorectal cancer are associated with poor prognosis but do not preclude benefit from oxaliplatin or irinotecan: results from the MRC FOCUS trial. J Clin Oncol 27:5931-5937. 10.1200/JCO.2009.22.4295

Roth AD, Tejpar S, Delorenzi M, Yan P, Fiocca R, Klingbiel D, Dietrich D, Biesmans B, Bodoky G, Barone C, Aranda E, Nordlinger B, Cisar L, Labianca R, Cunningham D, Van Cutsem E, and Bosman F. 2010. Prognostic role of KRAS and BRAF in stage II and III resected colon cancer: results of the translational study on the PETACC-3, EORTC 40993, SAKK 60-00 trial. J Clin Oncol 28:466-474. 10.1200/JCO.2009.23.3452

Salem ME, Weinberg BA, Xiu J, El-Deiry WS, Hwang JJ, Gatalica Z, Philip PA, Shields AF, Lenz HJ, and Marshall JL. 2017. Comparative molecular analyses of left-sided colon, right-sided colon, and rectal cancers. Oncotarget 8:86356-86368. 10.18632/oncotarget.21169

Sanz-Garcia E, Argiles G, Elez E, and Tabernero J. 2017. BRAF mutant colorectal cancer: prognosis, treatment, and new perspectives. Ann Oncol 28:2648-2657. 10.1093/annonc/mdx401

Shen W, Sakamoto N, and Yang L. 2016. Melanoma-specific mortality and competing mortality in patients with nonmetastatic malignant melanoma: a population-based analysis. BMC Cancer 16:413. 10.1186/s12885-0162438-3

Society. AC. 2017. Colorectal Cancer Facts \& Figures 2017-2019.

. Atlanta: American Cancer Society.

Souglakos J, Philips J, Wang R, Marwah S, Silver M, Tzardi M, Silver J, Ogino S, Hooshmand S, Kwak E, Freed E, Meyerhardt JA, Saridaki Z, Georgoulias V, Finkelstein D, Fuchs CS, Kulke MH, and Shivdasani RA. 2009. Prognostic and predictive value of common mutations for treatment response and survival in patients with metastatic colorectal cancer. Br J Cancer 101:465-472. 10.1038/sj.bjc.6605164

Sperlich A, Balmert A, Doll D, Bauer S, Franke F, Keller G, Wilhelm D, Mur A, Respondek M, Friess H, Nitsche U, and Janssen KP. 2018. Genetic and immunological biomarkers predict metastatic disease recurrence in stage III colon cancer. BMC Cancer 18:998. 10.1186/s12885-018-4940-2

Taieb J, Shi Q, Pederson L, Alberts S, Wolmark N, Van Cutsem E, de Gramont A, Kerr R, Grothey A, Lonardi S, Yoshino T, Yothers G, Sinicrope FA, Zaanan A, and Andre T. 2019. Prognosis of microsatellite instability and/or mismatch repair deficiency stage III colon cancer patients after disease recurrence following adjuvant treatment: results of an ACCENT pooled analysis of seven studies. Ann Oncol 30:1466-1471. 10.1093/annonc/mdz208

Tan C, and Du X. 2012. KRAS mutation testing in metastatic colorectal cancer. World J Gastroenterol 18:5171-5180. 10.3748/wjg.v18.i37.5171

van Brummelen EMJ, de Boer A, Beijnen JH, and Schellens JHM. 2017. BRAF Mutations as Predictive Biomarker for Response to Anti-EGFR Monoclonal Antibodies. Oncologist 22:864-872. 10.1634/theoncologist.2017-0031

van Eeghen EE, Bakker SD, van Bochove A, and Loffeld RJ. 2015. Impact of age and comorbidity on survival in 
colorectal cancer. J Gastrointest Oncol 6:605-612. 10.3978/j.issn.2078-6891.2015.070

Venook AP, Niedzwiecki D, Lenz HJ, Innocenti F, Fruth B, Meyerhardt JA, Schrag D, Greene C, O'Neil BH, Atkins JN, Berry S, Polite BN, O'Reilly EM, Goldberg RM, Hochster HS, Schilsky RL, Bertagnolli MM, El-Khoueiry AB, Watson P, Benson AB, 3rd, Mulkerin DL, Mayer RJ, and Blanke C. 2017. Effect of First-Line Chemotherapy Combined With Cetuximab or Bevacizumab on Overall Survival in Patients With KRAS Wild-Type Advanced or Metastatic Colorectal Cancer: A Randomized Clinical Trial. JAMA 317:2392-2401. 10.1001/jama.2017.7105

Yang L, Shen W, and Sakamoto N. 2013. Population-based study evaluating and predicting the probability of death resulting from thyroid cancer and other causes among patients with thyroid cancer. J Clin Oncol 31:468474. 10.1200/JCO.2012.42.4457

Zhang Z. 2017. Survival analysis in the presence of competing risks. Ann Transl Med 5:47. 10.21037/atm.2016.08.62

\section{Figure legends}

Figure 1. CSS of CRC patients with different stages according to KRAS mutation status by CIF plot

CIF plots of KRAS mutation status and the prognosis of CRC in overall population (a) and stage I-IV CRC patients (b-e).

Figure 2. CSS of CRC patients with differed location according to KRAS mutation status by CIF plot

CIF plots of KRAS mutation status and the prognosis of CRC in locations of unknown (a), left colon (b), right colon (c) and rectum (d). NOS, not otherwise specified.

Figure 3. Nomogram for predicting 1-year, 2-year and 3-year CSS of CRC patients who had KRAS testing

The nomogram is used by summing the points identified on the top scale for each independent variable and drawing a vertical line from the total points scale to the 1-year, 2-year and 3-year CSS to obtain the probability of survival. The total points projected to the bottom scale indicate the \% probability of the 3-year survival. Age: $2=20-29$ years, 3 $=30-39$ years, $4=40-49$ years, $5=50-59$ years, $6=60-69$ years and $7=70-79$ years; Race: 1 = Caucasian, 2 = African American, $3=$ Other race and $\mathrm{N}=$ Unknown race; Tumor size: 2 = "0cm-2cm", $4=$ " $2 \mathrm{~cm}-4 \mathrm{~cm}$ ", $6=$ " $4-6 \mathrm{~cm}$ ", $>6$ = ">6cm", $N$ = Unknown size; Tumor stage, $0=0$ stage (Tumor in situ), $1=$ I stage, $2=I I$ stage, $3=$ III stage, $4=$ IV stage and $\mathrm{N}=$ Unknown stage; NO. Nodes, the number of positive regional lymph nodes; KRAS mutation status: $0=$ Wild type and $1=$ Mutation; Chemotherapy, $0=$ none/unknown and 1 = yes; Radiotherapy, 0 = none/unknown or refused, 1 = beam radiation or combination 
399 of beam with implants or isotopes or radiation with method or source not specified or 400 radioactive implants or radioisotopes and $\mathrm{N}=$ Recommended, unknown if administered.

401

402 Figure 4. Calibration curves for Cox-based and $\mathrm{SH}$ based nomograms

403 a-c: The calibration plots for predicting 1-year, 2-year and 3-year CSS of CRC patients, 404 the $x$-axis was the nomogram predicted risk, and the $y$-axis was the actual risk; $d$ : The 405 time dependent (1-year, 2-year and 3-year) AUC plots for SH-based nomogram. 406

407 Supplemental Fig. S1. The median survival time of different groups from SEER database 408 after screening

409 The current study included with 2010-2012 group (arrow), which exhibited similar median 410 survival time as 2010 group.

411

412 Supplemental Fig. S2. KRAS testing rates by years of metastatic CRC and non413 metastatic CRC according to SEER. 


\section{Table $\mathbf{1}$ (on next page)}

The characteristic of each included variables in KRAS MT and KRAS WT patients

KRAS MT, KRAS mutant; KRAS WT, KRAS wild type; the widowed or single (never married or having a domestic partner) or divorced or separated patients was defined as unmarried; Tis, Tumor in situ; $p$ value referred to the difference between MT and WT KRAS patients; the significant $p$ values were bolded. 
1 Table 1. The characteristic of each included variable in KRAS MT and KRAS WT patients

\begin{tabular}{|c|c|c|c|c|c|}
\hline \multirow[b]{2}{*}{ Characteristics } & \multicolumn{2}{|l|}{ KRAS MT } & \multicolumn{2}{|c|}{ KRAS WT } & \multirow[b]{2}{*}{$p$ value } \\
\hline & No. of patients & $\%$ & $\begin{array}{l}\text { No. of } \\
\text { patients }\end{array}$ & $\%$ & \\
\hline Age & & & & & 0.045 \\
\hline$<29$ & 38 & $1.05 \%$ & 70 & $1.30 \%$ & \\
\hline $30-39$ & 145 & $4.01 \%$ & 246 & $4.58 \%$ & \\
\hline $40-49$ & 503 & $13.91 \%$ & 738 & $13.75 \%$ & \\
\hline $50-59$ & 820 & $22.68 \%$ & 1,261 & $23.50 \%$ & \\
\hline $60-69$ & 992 & $27.43 \%$ & 1,435 & $26.74 \%$ & \\
\hline $70-79$ & 749 & $20.71 \%$ & 995 & $18.54 \%$ & \\
\hline$>=80$ & 369 & $10.20 \%$ & 622 & $11.59 \%$ & \\
\hline Sex & & & & & 0.023 \\
\hline Female & 1,697 & $46.93 \%$ & 2,387 & $44.48 \%$ & \\
\hline Male & 1,919 & $53.07 \%$ & 2,980 & $55.52 \%$ & \\
\hline Race & & & & & $<0.001$ \\
\hline White & 2,757 & $76.24 \%$ & 4,264 & $79.45 \%$ & \\
\hline African Americans & 518 & $14.33 \%$ & 598 & $11.14 \%$ & \\
\hline Others & 328 & $9.07 \%$ & 490 & $9.13 \%$ & \\
\hline Unknown & 13 & $0.36 \%$ & 15 & $0.28 \%$ & \\
\hline Location & & & & & $<0.001$ \\
\hline Left & 1,208 & $33.41 \%$ & 2,268 & $42.26 \%$ & \\
\hline NOS & 97 & $2.68 \%$ & 160 & $2.98 \%$ & \\
\hline Rectum & 665 & $18.39 \%$ & 992 & $18.48 \%$ & \\
\hline right & 1,646 & $45.52 \%$ & 1,947 & $36.28 \%$ & \\
\hline Tumor size & & & & & 0.086 \\
\hline$<=2 \mathrm{~cm}$ & 399 & $11.03 \%$ & 647 & $12.06 \%$ & \\
\hline$>6$ & 674 & $18.64 \%$ & 997 & $18.58 \%$ & \\
\hline 2 to 4 & 855 & $23.64 \%$ & 1,363 & $25.40 \%$ & \\
\hline 4 to 6 & 1,054 & $29.15 \%$ & 1,476 & $27.50 \%$ & \\
\hline $\mathrm{N}$ & 634 & $17.53 \%$ & 884 & $16.47 \%$ & \\
\hline Surgery & & & & & 0.005 \\
\hline No & 816 & $22.57 \%$ & 1,079 & $20.10 \%$ & \\
\hline Yes & 2,800 & $77.43 \%$ & 4,288 & $79.90 \%$ & \\
\hline Stage & & & & & $<0.001$ \\
\hline $0 /($ Tis $)$ & 13 & $0.36 \%$ & 12 & $0.22 \%$ & \\
\hline I & 202 & $5.59 \%$ & 384 & $7.15 \%$ & \\
\hline II & 461 & $12.75 \%$ & 817 & $15.22 \%$ & \\
\hline III & 887 & $24.53 \%$ & 1,494 & $27.84 \%$ & \\
\hline IV & 2,020 & $55.86 \%$ & 2,615 & $48.72 \%$ & \\
\hline
\end{tabular}




\begin{tabular}{|c|c|c|c|c|c|}
\hline Unknown & 33 & $0.91 \%$ & 45 & $0.84 \%$ & \\
\hline Grade & & & & & $<0.001$ \\
\hline Low grade (I \& II) & 2,502 & $69.19 \%$ & 3,490 & $65.03 \%$ & \\
\hline High grade (III \& IV) & 712 & $19.69 \%$ & 1,370 & $25.53 \%$ & \\
\hline NOS & 402 & $11.12 \%$ & 507 & $9.45 \%$ & \\
\hline $\begin{array}{l}\text { Regional nodes } \\
\text { positive }\end{array}$ & & & & & 0.025 \\
\hline$>=10$ & 1,196 & $33.08 \%$ & 1,726 & $32.16 \%$ & \\
\hline 0 & 903 & $24.97 \%$ & 1,485 & $27.67 \%$ & \\
\hline 1 to 3 & 880 & $24.34 \%$ & 1,212 & $22.58 \%$ & \\
\hline 4 to 9 & 637 & $17.62 \%$ & 944 & $17.59 \%$ & \\
\hline Radiotherapy & & & & & 0.383 \\
\hline No & 3,058 & $84.57 \%$ & 4,576 & $85.26 \%$ & \\
\hline Yes & 535 & $14.80 \%$ & 772 & $14.38 \%$ & \\
\hline Unknown & 23 & $0.64 \%$ & 19 & $0.35 \%$ & \\
\hline Chemotherapy & & & & & 0.002 \\
\hline No & 924 & $25.55 \%$ & 1,534 & $28.58 \%$ & \\
\hline Yes & 2,692 & $74.45 \%$ & 3,833 & $71.42 \%$ & \\
\hline Marital status & & & & & 0.237 \\
\hline Married & 1,988 & $54.98 \%$ & 2,958 & $55.11 \%$ & \\
\hline Unmarried & 1,464 & $40.49 \%$ & 2,204 & $41.07 \%$ & \\
\hline Unknown & 164 & $4.54 \%$ & 205 & $3.82 \%$ & \\
\hline
\end{tabular}

2 KRAS MT, KRAS mutant; KRAS WT, KRAS wild type; the widowed or single (never married or having a

3 domestic partner) or divorced or separated patients was defined as unmarried; Tis, Tumor in situ; $p$ value 4 referred to the difference between MT and WT KRAS patients; the significant $p$ values were bolded. 


\section{Table 2 (on next page)}

Subgroup analysis of KRAS mutation status and the prognosis of CRC patients.

KRAS MT, KRAS mutant; KRAS WT, KRAS wild type; HR, Hazard ratios; 95\%Cl, 95\%confidence intervals; the significant results were bolded. 
1 Table 2. Subgroup analysis of KRAS mutation status and the prognosis of CRC

2 patients.

\begin{tabular}{lll}
\hline \multirow{2}{*}{ Group } & & \multicolumn{2}{l}{ KRAS MT vs. KRAS WT } \\
\cline { 3 - 3 } & Patients amount & Multivariate SH model \\
\cline { 3 - 3 } & & $\mathbf{H R}(95 \% \mathrm{CI})$ \\
\hline All & 8,983 & $1.04, \mathbf{1 . 1 7})$ \\
Stage I & 586 & $1.30(0.62-2.70)$ \\
Stage II & 1,278 & $\mathbf{1 . 2 8}(\mathbf{1 . 0 9 - 1 . 4 9 )}$ \\
Stage III & 2,381 & $\mathbf{1 . 1 4}(\mathbf{1 . 0 6 - 1 . 2 3 )}$ \\
Stage IV & 4,635 & $1.01(0.69,1.49)$ \\
Unknown location & 257 & $\mathbf{1 . 2 8}(\mathbf{1 . 1 5}, \mathbf{1 . 4 2})$ \\
Left & 3,476 & $1.07(0.97,1.19)$ \\
Right & 3,593 & $\mathbf{1 . 2 3}(\mathbf{1 . 0 7}, \mathbf{1 . 4 3})$ \\
Rectum & 1,657 & .
\end{tabular}

3 KRAS MT, KRAS mutant; KRAS WT, KRAS wild type; HR, Hazard ratios; $95 \% \mathrm{Cl}, 95 \%$ confidence

4 intervals; the significant results were bolded. 


\section{Table 3 (on next page)}

Multivariate SH analyses of each variables in KRAS MT and WT patients

KRAS MT, KRAS mutant; KRAS WT, KRAS wild type; HR, Hazard ratios; 95\%Cl, 95\%confidence intervals; the widowed or single (never married or having a domestic partner) or divorced or separated patients was defined as unmarried; Tis, Tumor in situ; significant results were bolded. 
1 Table 3. Multivariate SH analyses of each variables in KRAS MT and WT patients

\begin{tabular}{|c|c|c|}
\hline \multirow{3}{*}{ Characteristics } & \multicolumn{2}{|c|}{ SH model } \\
\hline & KRAS MT & KRAS WT \\
\hline & $\mathrm{HR}(95 \% \mathrm{Cl})$ & $\mathrm{HR}(95 \% \mathrm{Cl})$ \\
\hline \multicolumn{3}{|l|}{ Age } \\
\hline$<29$ & Reference & Reference \\
\hline $30-39$ & $0.58(0.36,0.94)$ & $1.19(0.85,1.66)$ \\
\hline $40-49$ & $0.71(0.46,1.11)$ & $1.05(0.77,1.44)$ \\
\hline $50-59$ & $0.72(0.46,1.13)$ & $1.11(0.82,1.51)$ \\
\hline $60-69$ & $0.59(0.38,0.92)$ & $1.03(0.76,1.40)$ \\
\hline $70-79$ & $0.57(0.37,0.90)$ & $0.99(0.72,1.36)$ \\
\hline$>=80$ & $0.58(0.36,0.94)$ & $0.93(0.66,1.31)$ \\
\hline \multicolumn{3}{|l|}{ Sex } \\
\hline Female & Reference & Reference \\
\hline Male & $1.07(0.98,1.18)$ & $0.97(0.89,1.06)$ \\
\hline \multicolumn{3}{|l|}{ Race } \\
\hline White & Reference & Reference \\
\hline African Americans & $1.16(1.02,1.33)$ & $1.03(0.90,1.18)$ \\
\hline Others & $1.02(0.87,1.20)$ & $1.10(0.96,1.27)$ \\
\hline Unknown & $0.35(0.05,2.57)$ & $0.99(0.29,3.37)$ \\
\hline \multicolumn{3}{|l|}{ Location } \\
\hline Left & Reference & Reference \\
\hline NOS & $0.97(0.71,1.31)$ & $1.15(0.90,1.48)$ \\
\hline Rectum & $0.87(0.75,1.01)$ & $0.95(0.83,1.08)$ \\
\hline right & $1.05(0.94,1.17)$ & $1.22(1.10,1.35)$ \\
\hline \multicolumn{3}{|l|}{ Tumor size } \\
\hline$<=2 \mathrm{~cm}$ & Reference & Reference \\
\hline $2-4 \mathrm{~cm}$ & $0.95(0.79,1.15)$ & $1.00(0.85,1.19)$ \\
\hline $4-6 \mathrm{~cm}$ & $1.00(0.83,1.20)$ & $1.10(0.94,1.29)$ \\
\hline$>6 \mathrm{~cm}$ & $1.06(0.87,1.28)$ & $1.25(1.05,1.48)$ \\
\hline $\mathrm{N}$ & $1.04(0.86,1.27)$ & $1.01(0.85,1.21)$ \\
\hline \multicolumn{3}{|l|}{ Surgery } \\
\hline No & Reference & Reference \\
\hline Yes & $0.77(0.65,0.91)$ & $0.86(0.75,0.995)$ \\
\hline \multicolumn{3}{|l|}{ Stage } \\
\hline $0 /($ Tis $)$ & Reference & Reference \\
\hline I & $1.55(0.22,10.88)$ & $1.07(0.14,8.48)$ \\
\hline II & $4.62(0.69,30.73)$ & $2.96(0.39,22.73)$ \\
\hline III & $6.96(1.06,45.70)$ & $4.08(0.54,30.82)$ \\
\hline IV & $18.90(2.88,123.93)$ & $11.31(1.50,85.33)$ \\
\hline Unknown & $5.52(0.78,39.14)$ & $5.01(0.64,39.46)$ \\
\hline
\end{tabular}




\begin{tabular}{lll}
$\begin{array}{l}\text { Grade } \\
\text { Low grade (I \& II) }\end{array}$ & Reference & Reference \\
High grade (III \& IV) & $\mathbf{1 . 3 5 ( 1 . 2 0 , 1 . 5 2 )}$ & $\mathbf{1 . 3 8}(\mathbf{1 . 2 5 , 1 . 5 3 )}$ \\
NOS & $0.95(0.81,1.10)$ & $1.07(0.93,1.23)$ \\
Regional nodes positive & & \\
>=10 & Reference & Reference \\
0 & $\mathbf{0 . 4 8}(\mathbf{0 . 3 9}, \mathbf{0 . 5 9})$ & $\mathbf{0 . 3 7}(\mathbf{0 . 3 1}, \mathbf{0 . 4 6})$ \\
1 to 3 & $\mathbf{0 . 6 1}(\mathbf{0 . 5 2}, \mathbf{0 . 7 3})$ & $\mathbf{0 . 5 0}(\mathbf{0 . 4 3}, \mathbf{0 . 5 7})$ \\
4 to 9 & $\mathbf{0 . 8 0}(\mathbf{0 . 6 8}, \mathbf{0 . 9 5})$ & $\mathbf{0 . 7 0}(\mathbf{0 . 6 1}, \mathbf{0 . 8 0})$ \\
Radiotherapy & & \\
No & Reference & Reference \\
Yes & $1.07(0.92,1.26)$ & $1.00(0.87,1.15)$ \\
Unknown & $1.04(0.56,1.92)$ & $0.45(0.19,1.09)$ \\
Chemotherapy & & \\
No & Reference & Reference \\
Yes & $\mathbf{0 . 7 7}(\mathbf{0 . 6 6 , 0 . 9 0 )}$ & $0.99(0.86,1.13)$ \\
Marital status & & \\
Married & Reference & Reference \\
Unmarried & $\mathbf{1 . 1 9 ( 1 . 0 8 , 1 . 3 2 )}$ & $\mathbf{1 . 1 1}(\mathbf{1 . 0 1}, \mathbf{1 . 2 1})$ \\
Unknown & $0.88(0.70,1.11)$ & $1.12(0.91,1.37)$ \\
\hline
\end{tabular}

2 KRAS MT, KRAS mutant; KRAS WT, KRAS wild type; HR, Hazard ratios; $95 \% \mathrm{Cl}, 95 \%$ confidence

3 intervals; the widowed or single (never married or having a domestic partner) or divorced or separated

4 patients was defined as unmarried; Tis, Tumor in situ; significant results were bolded. 


\section{Table 4 (on next page)}

Variable selection: Estimated coefficients (SES) for the current SH model

LASSO, least absolute shrinkage and selection operator; SCAD, smoothly clipped absolute deviation (SCAD); MCP, measure-correlate-predict (MCP). 
1 Table 4. Variable selection: Estimated coefficients (SEs) for the current SH model

\begin{tabular}{llll}
\hline Characteristics & LASSO & SCAD & MCP \\
\hline Age & -0.022 & -0.025 & -0.028 \\
Sex & 0.000 & 0.000 & 0.000 \\
Race & 0.000 & 0.000 & 0.000 \\
Location & -0.032 & -0.041 & -0.044 \\
Surgery & -0.069 & 0.000 & 0.000 \\
Tumor size & 0.017 & 0.027 & 0.025 \\
Tumor stage & 0.226 & 0.233 & 0.233 \\
Grade & 0.000 & 0.000 & 0.000 \\
Regional nodes positive & 0.097 & 0.105 & 0.105 \\
KRAS status & 0.13 & 0.182 & 0.183 \\
Chemotherapy & 0.314 & 0.384 & 0.383 \\
Radiotherapy & -0.061 & -0.095 & -0.095 \\
Marital status & 0.000 & 0.000 & 0.000 \\
\hline
\end{tabular}

2 LASSO, least absolute shrinkage and selection operator; SCAD, smoothly clipped absolute deviation

3 (SCAD); MCP, measure-correlate-predict (MCP).

4 
Figure 1

\section{CSS of CRC patients with different stages according to KRAS status by CIF plot}

CIF plots of KRAS status and the prognosis of CRC in overall population (a) and stage I-IV CRC patients (b-e).
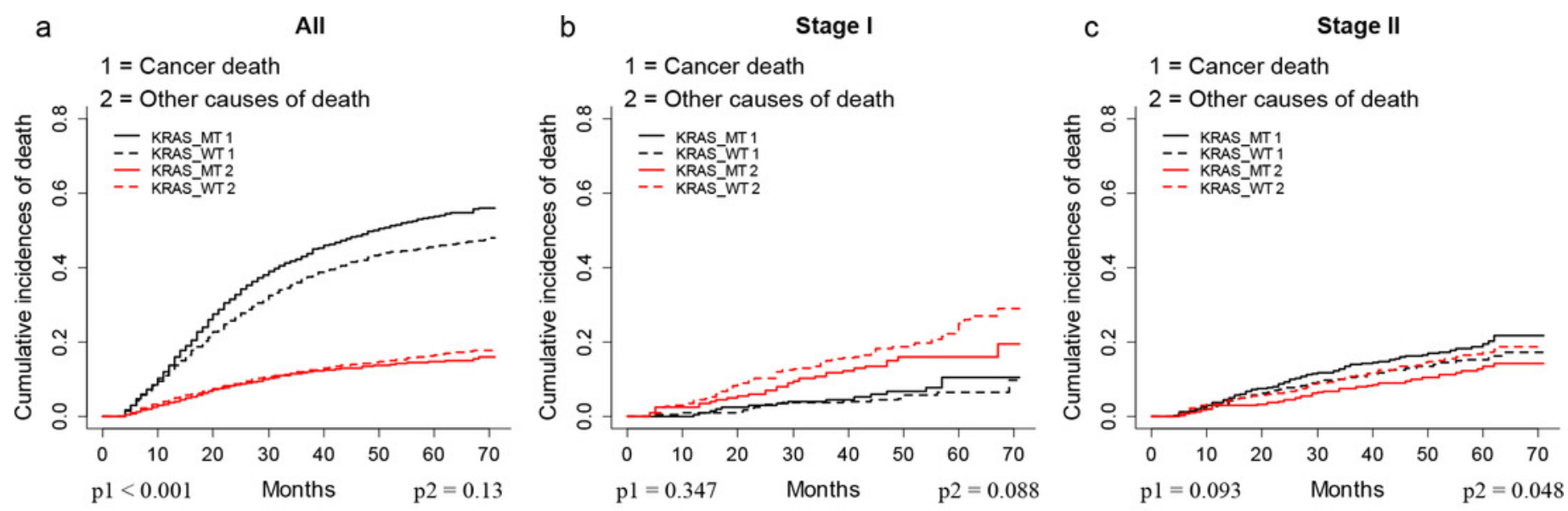

d

Stage III

$1=$ Cancer death
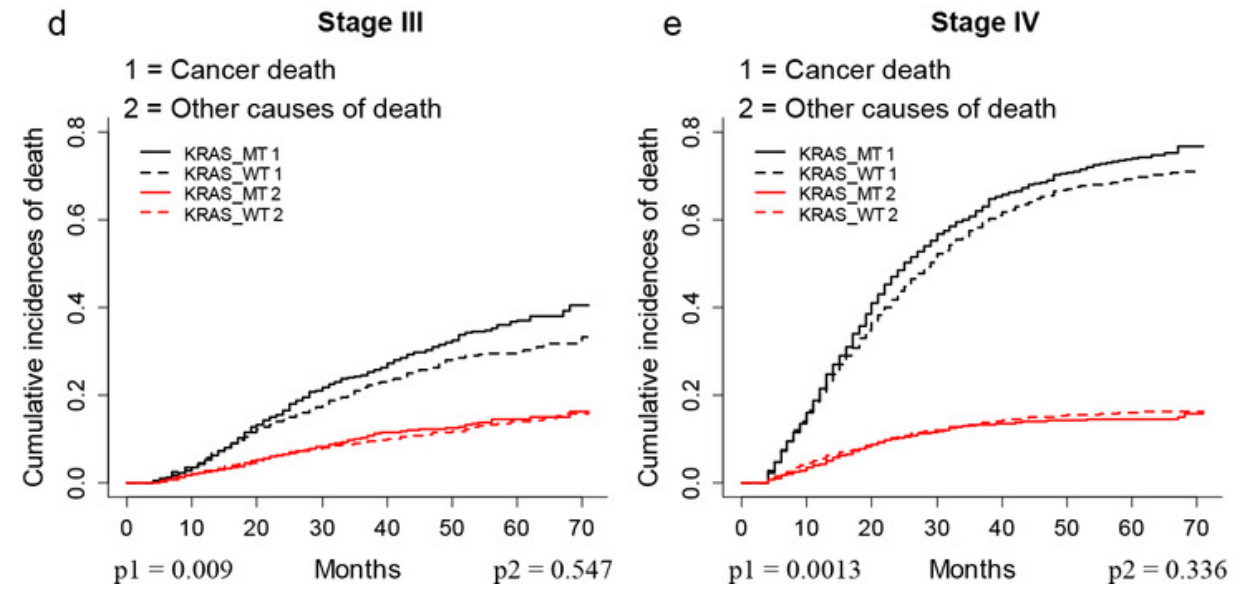
Figure 2

CSS of CRC patients with differed location according to KRAS status by CIF plot

CIF plots of KRAS status and the prognosis of CRC in locations of unknown (a), left colon (b), right colon (c) and rectum (d). NOS, not otherwise specified.

a

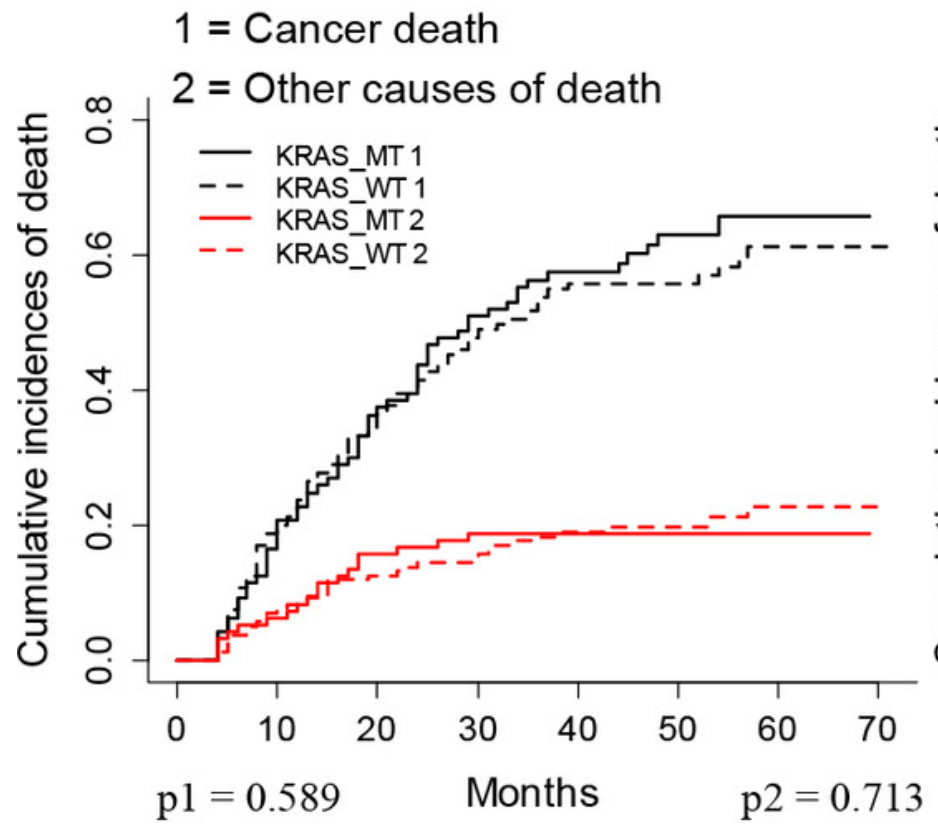

C

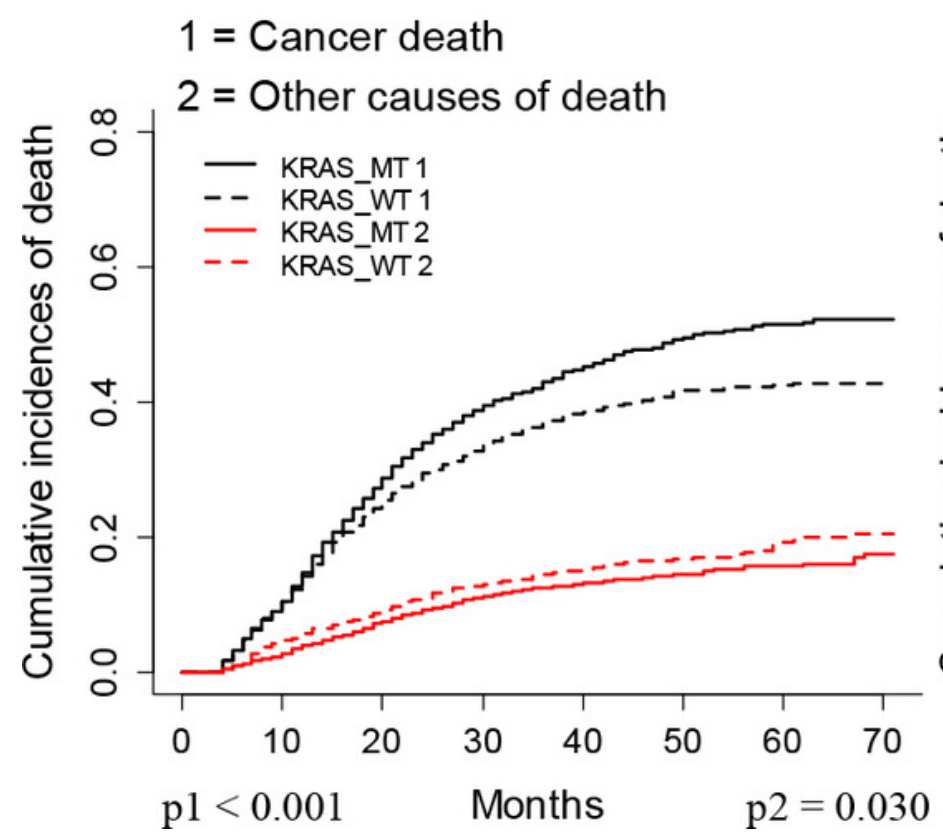

b

Left

1 = Cancer death

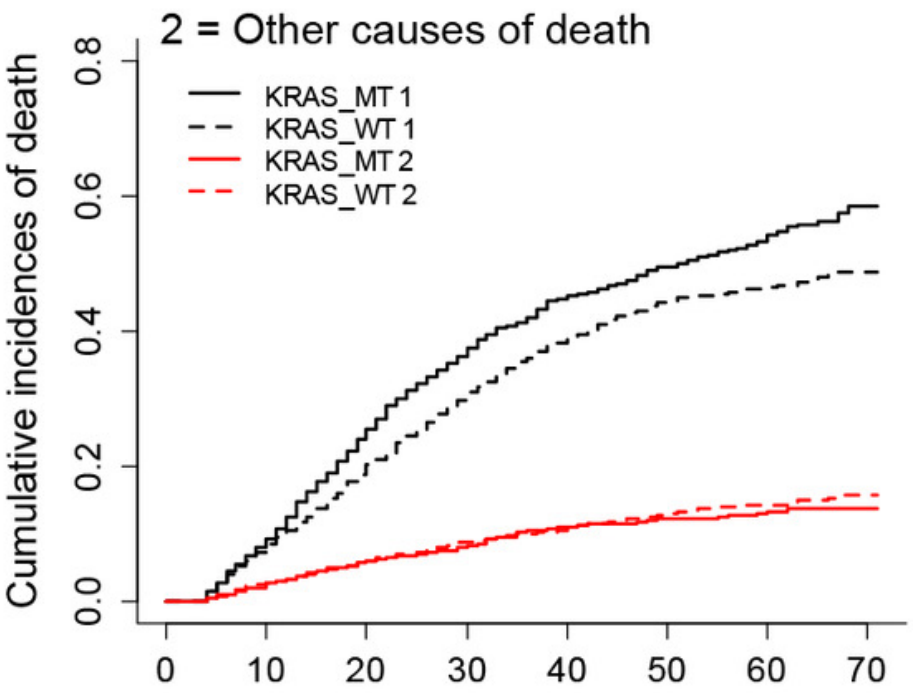

p1 $<0.001$

d

p2 $=0.496$

\section{Rectum}

$1=$ Cancer death

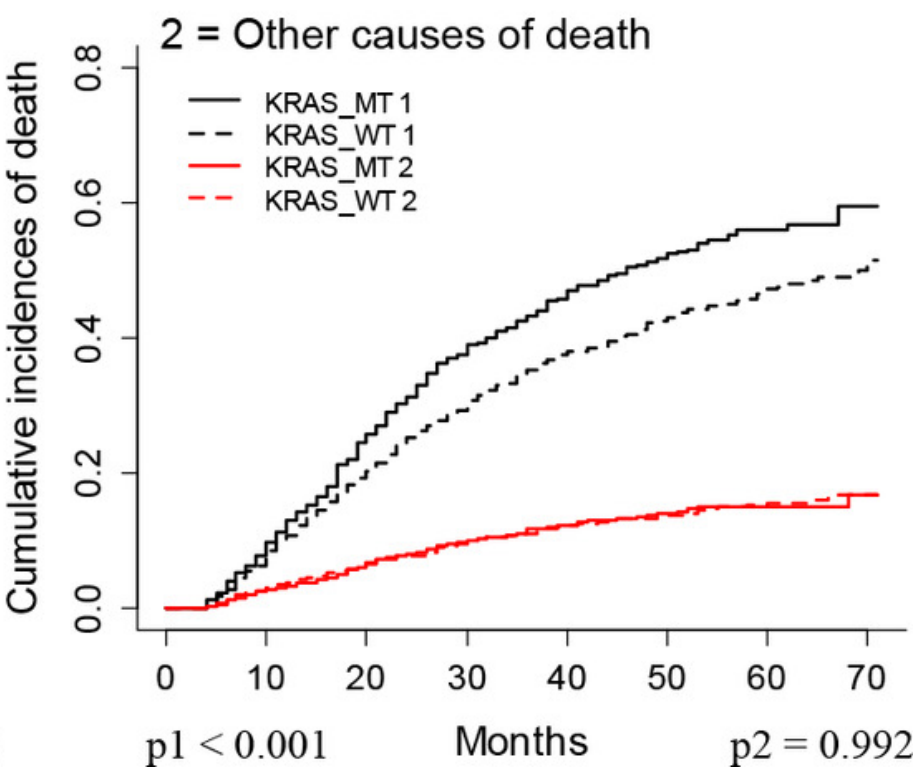




\section{Figure 3}

Nomogram for predicting 1-year, 2-year and 3-year CSS of CRC patients who had KRAS testing

The nomogram is used by summing the points identified on the top scale for each independent variable and drawing a vertical line from the total points scale to the 1-year, 2year and 3-year CSS to obtain the probability of survival. The total points projected to the bottom scale indicate the $\%$ probability of the 3-year survival. Age: $2=20-29$ years, $3=$ 30-39 years, $4=40-49$ years, $5=50-59$ years, $6=60-69$ years and $7=70-79$ years; Race: 1 = Caucasian, 2 = African American, $3=$ Other race and $\mathrm{N}=$ Unknown race; Tumor size: 2 = "0 $\mathrm{cm}-2 \mathrm{~cm}$ ", $4=$ " $2 \mathrm{~cm}-4 \mathrm{~cm}$ ", $6=$ "4- $6 \mathrm{~cm}$ ", $>6=$ "> $>6 \mathrm{~cm}$ ", $\mathrm{N}=$ Unknown size; Tumor stage, 0 $=0$ stage (Tumor in situ), $1=$ I stage, $2=I I$ stage, $3=I I I$ stage, $4=I V$ stage and $\mathrm{N}=$ Unknown stage; NO. Nodes, the number of positive regional lymph nodes; KRAS status: $0=$ Wild type and $1=$ Mutation; Chemotherapy, $0=$ none/unknown and $1=$ yes; Radiotherapy, 0 $=$ none/unknown or refused, $1=$ beam radiation or combination of beam with implants or isotopes or radiation with method or source not specified or radioactive implants or radioisotopes and $\mathrm{N}=$ Recommended, unknown if administered. 


\section{SH model}

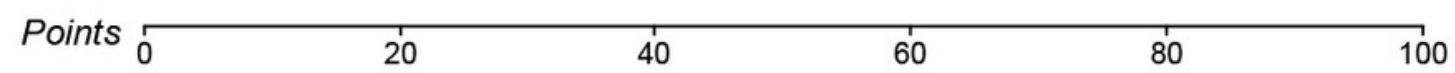

Age

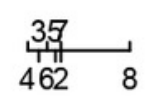

Location $^{* \star *}$

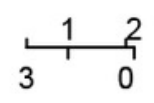

Tumor_size***

$$
\underset{46 \rightarrow 6}{2+1}
$$

Tumor_stage ${ }^{* * *}$

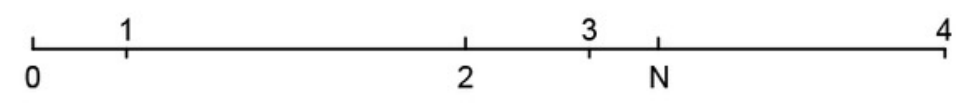

NO.Nodes ${ }^{* * *}$

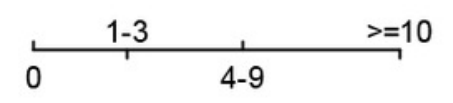

KRAS_status***

$$
\stackrel{1}{0}
$$

Chemotherapy***

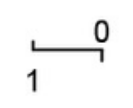

Radiotherapy

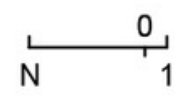

\section{Total-points-to-outcome nomogram:}

\begin{tabular}{|c|c|c|c|c|c|c|c|c|c|c|c|c|c|}
\hline $\operatorname{Pr}(T<$ & 0.005 & 0.01 & 0.01 & 0.02 & $0.03 \quad 0.04$ & 0.0 & & 0.1 & 0.15 & 0.25 & 0.35 & 0.45 & 0.55 \\
\hline$(T<24)_{0.01}$ & 0.0150 .02 & 0.03 & 0.04 & 0.06 & 0.1 & 0.15 & 0.2 & 0.3 & 0.4 & $0.5 \quad 0.6$ & 0.7 & 0.8 & 0.9 \\
\hline & & 04 & 0.06 & 0.1 & $0.15 \quad 0.2$ & & 0.3 & 0.4 & $0.5 \quad 0.6$ & 0.7 & 0.85 & 0.930 & .97 \\
\hline
\end{tabular}

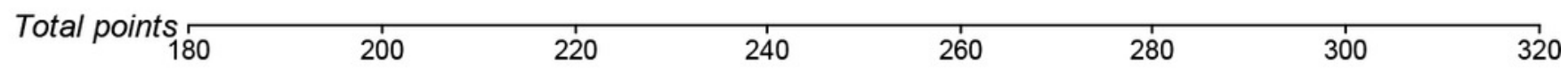


Figure 4

Calibration curves for Cox-based and SH based nomograms

a-c: The calibration plots for predicting 1-year, 2-year and 3-year CSS of CRC patients; d: The AUC plots for SH-based nomogram. 
a

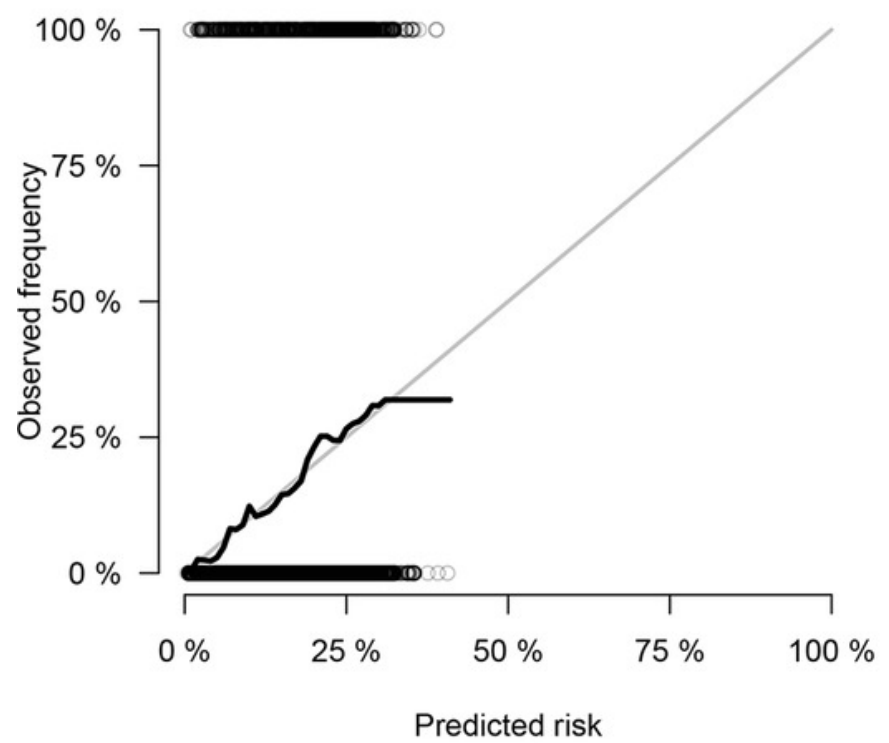

C

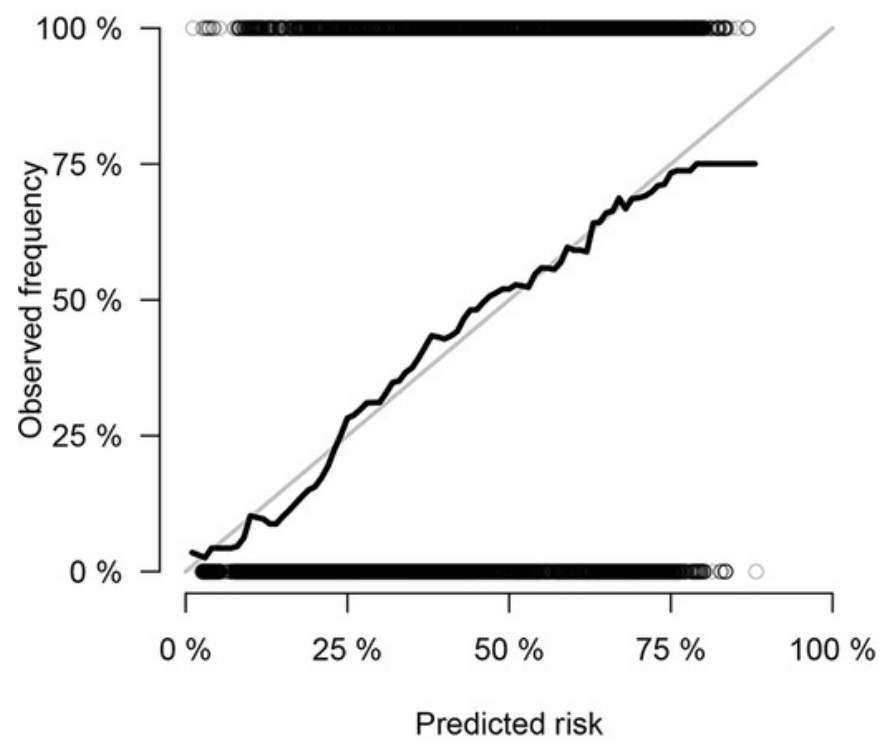

b

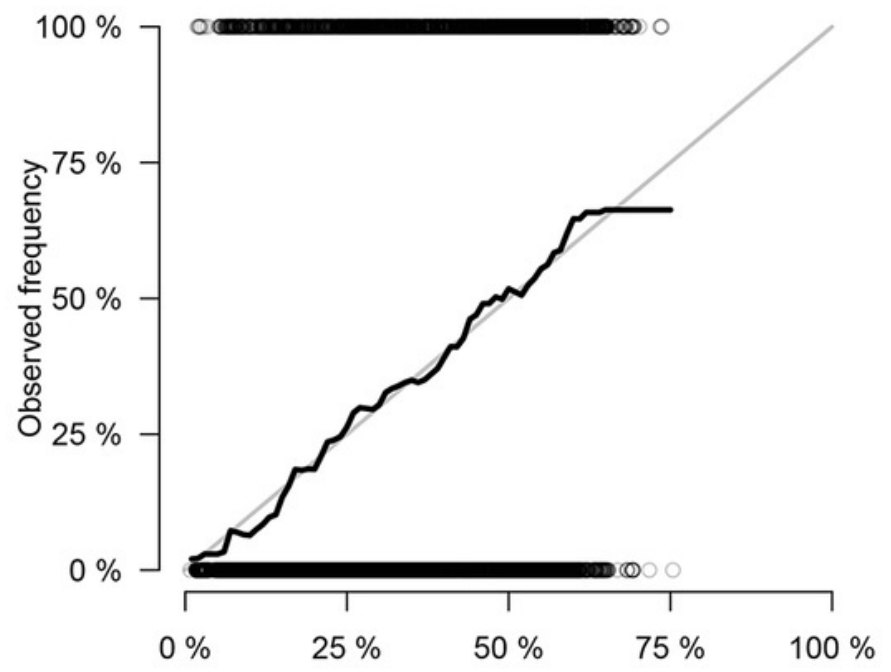

Predicted risk

d

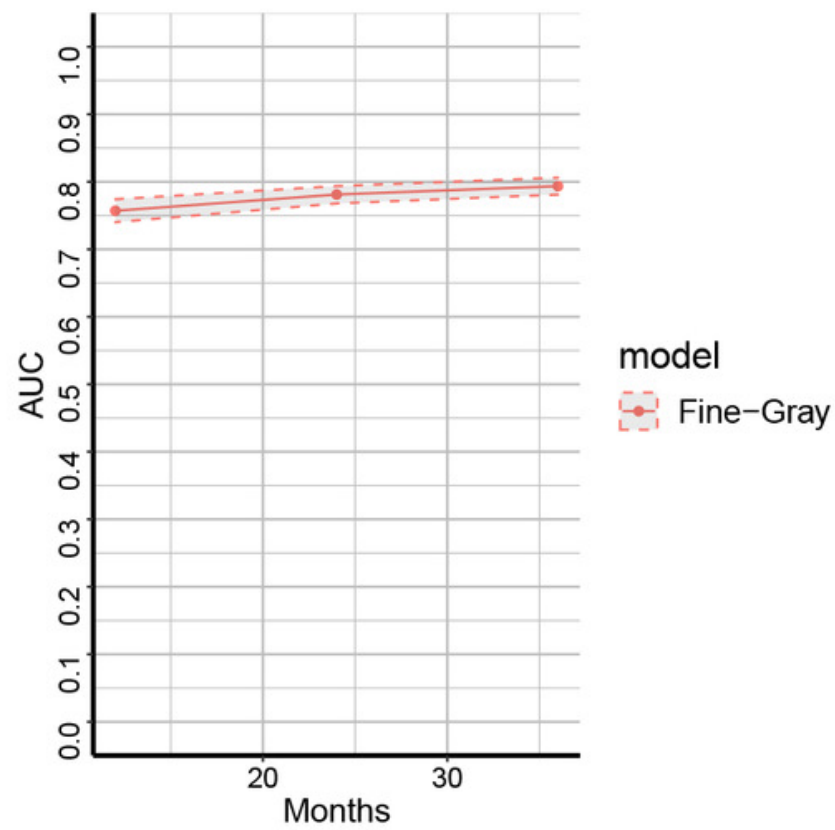

